\title{
Regional and whole-body imaging in pediatric oncology
}

\author{
Hyun Woo Goo
}

Received: 5 December 2010 /Accepted: 1 January 2011

(C) Springer-Verlag 2011

\begin{abstract}
The goals of tumor imaging include tumor detection, tumor characterization and differential diagnosis, imaging-guided biopsy, evaluation of tumor extent and staging, assessment of treatment responses, and surveillance for residual tumor or tumor recurrence. In clinical practice, various combinations of imaging modalities are used to achieve these goals. Recently introduced tumor imaging methods, such as diffusion MRI, perfusion MRI, whole-body MRI, and positron emission tomography (PET$\mathrm{CT}$ ), have shown promising results. Depending on tumor type and management plan, imaging protocols for children should be individually optimized to achieve the shortest examination time, the highest image quality, the lowest risk, and maximum clinical benefits. In this article, the roles of regional and whole-body tumor imaging will be reviewed, and several important issues related to recent technical developments will be discussed.
\end{abstract}

Keywords Child · Neoplasm · US · CT · MRI

The roles of tumor imaging include tumor detection, tumor characterization and differential diagnosis, imaging-guided

Disclaimer The supplement this article is part of is not sponsored by the industry. Dr. Goo has no financial interests, investigational or off-label uses to disclose.

Electronic supplementary material The online version of this article (doi:10.1007/s00247-011-2050-2) contains supplementary material, which is available to authorized users.

H. W. Goo $(\bowtie)$

Department of Radiology and Research Institute of Radiology,

Asan Medical Center, University of Ulsan College of Medicine,

86 Asanbyeongwon-gil, Songpa-gu,

Seoul 138-736, South Korea

e-mail: hwgoo@amc.seoul.kr biopsy, evaluation of tumor extent and staging, assessment of treatment responses, and surveillance for residual tumor or tumor recurrence. In clinical practice, various combinations of imaging modalities, including radiography, sonography, CT, MRI, and scintigraphy, have been used for the accurate assessment of pediatric tumors [1]. Imaging protocols should be individualized depending on tumor type and management plan, and should be updated as new imaging techniques are introduced. Recently developed techniques include contrast-enhanced sonography, diffusion MRI, perfusion MRI, whole-body MRI, and positron emission tomography (PET)-CT [2]. In this paper, the roles of regional and whole-body tumor imaging, excluding scintigraphy, will be reviewed, and several important issues related to recent technical developments will be discussed.

\section{Radiography}

Radiography can be used to identify high-contrast lesions, typically in lung and bone; calcification and/or ossification; and abnormal gas. Because the diagnostic yield is generally not very high, further evaluation with cross-sectional imaging modalities is usually required. Radiography is generally performed only in areas of clinical interest. Whole-body radiography is rarely conducted, with some exceptions, e.g., skeletal survey in Langerhans cell histiocytosis [3, 4].

\section{Sonography}

Gray-scale and Doppler sonography is used frequently as an initial imaging method for abdominal, pelvic, and soft- 
Fig. 1 a, b Mediastinal primitive neuroectodermal tumor in a newborn boy. a Gray-scale sonography of the chest shows an isoechoic solid lesion $(M)$ next to the normal thymus $(T)$. The lesion was initially incorrectly interpreted as thymic hemorrhage. b Color Dopplersonography performed the next day reveals dilated, disorganized vessels in the mediastinal lesion, suggesting a malignant tumor. This finding led to prompt biopsy
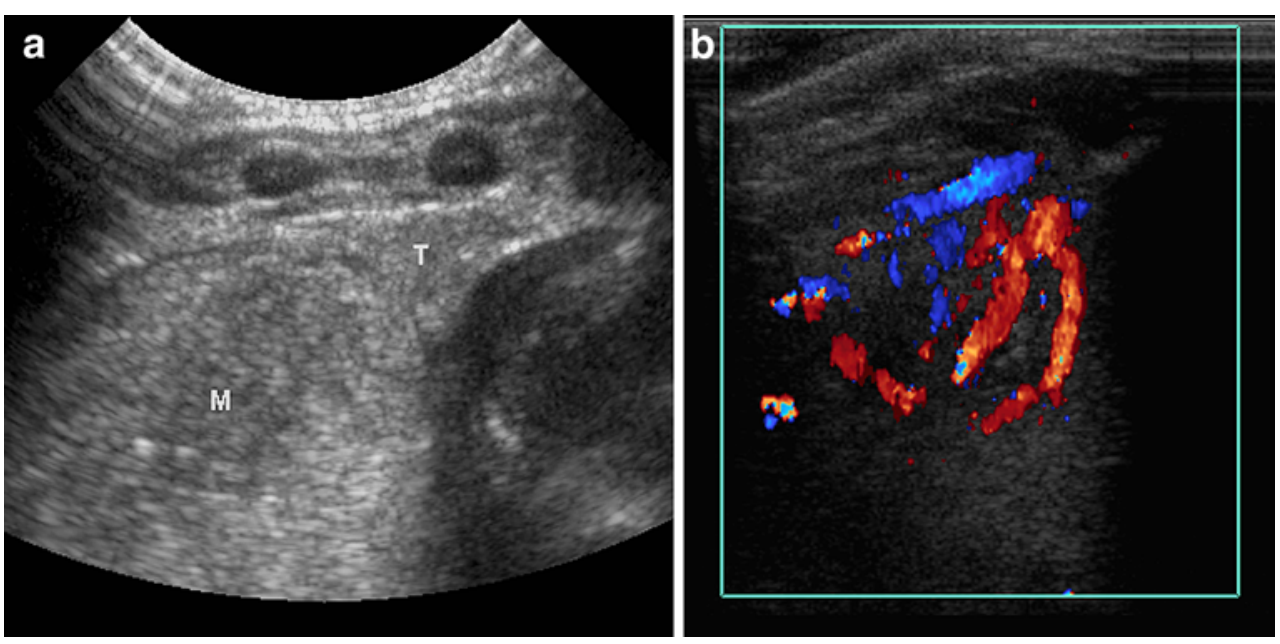

tissue tumors. In addition to localizing and characterizing (e.g., cystic, solid, or mixed) tumors, sonography may be used to evaluate loco-regional associated abnormalities, such as neovascularity (Fig. 1), vascular encasement, and lymphadenopathy. Real-time sonographic sliding signs are greatly helpful in localizing tumors, especially in pediatric patients with a paucity of intervisceral fat [5]. If necessary, a portable sonographic unit may be used to examine patients in the intensive care unit or the emergency department. However, sonography is limited by operator dependency and by restricted fields of view. Recent technical developments in sonography that may have additional value in tumor imaging include three-dimensional [6], extended field-of-view [7, 8] (Fig. 2), and contrast-enhanced sonography [2].

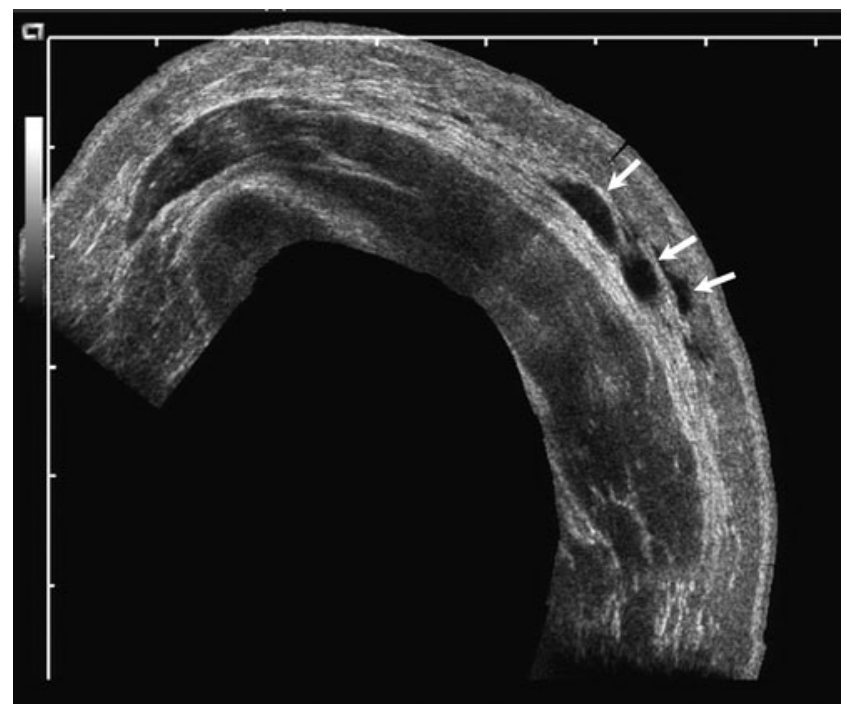

Fig. 2 Extended field-of-view sonography shows variable-size cystic elements (arrows) of a lymphangioma in the subcutaneous layer of the left upper arm in a 6-year-old girl

\section{CT}

CT is commonly used for initial and follow-up examinations. Because of the short scan time, CT can be used in severely ill patients. In addition, multi-slice CT with near-isotropic high spatial resolution can yield goodquality multi-planar reformatted images (Fig. 3) as well as three-dimensional images that may improve the accuracy of evaluation of the tumor extent [9-11]. Multi-slice chest CT facilitates the detection of lung nodules by reducing motion artifacts. The use of partial reconstruction [12] or electrocardiogram (ECG)-triggered data acquisition [13] can substantially reduce cardiac pulsation artifacts on chest $\mathrm{CT}$, resulting in improved detection of small lung nodules (Fig. 4). Recently, combined ECG- and respiratory-triggered data acquisition has been utilized in free-breathing children to eliminate cardiac and respiratory motion artifacts on chest CT [14]. Despite the improved detection of lung nodules when CT is used, recent studies have shown that single or multislice spiral chest CT could not adequately distinguish malignant from benign nodules $[15,16]$. Prone CT scanning is useful for differentiating true posterior subpleural nodules from nodular atelectasis [17], a frequent diagnostic dilemma in children (Fig. 5). CT is fairly sensitive when used to detect calcification or fat in a tumor. The limitations of CT are associated with the use of ionizing radiation and iodinated contrast agents, as well as relatively inferior soft tissue contrast resolution. To minimize radiation exposure during diagnostic $\mathrm{CT}$, low-dose CT protocols should be appropriately tailored to age or body size $[18,19]$. A recent study [20] found that $40 \%$ of surveillance imaging studies using ionizing radiation, predominantly CT (94\%) and nuclear scintigraphy, were performed in children with low risk of recurrence and/or with no clear indication. These findings emphasize the need for guidelines for surveillance 
Fig. 3 a, b Ewing sarcoma of the liver in a 10 -year-old boy. a Axial abdominal CT shows a large, predominantly hypodense tumor involving the right lobe. The inferior vena cava (arrows) is compressed and displaced by the tumor and appears elongated in the anteroposterior direction. b Coronal reformatted abdominal CT image shows the spatial relationship between the tumor and adjacent structures such as the inferior vena cava (asterisks) and right kidney (arrows)
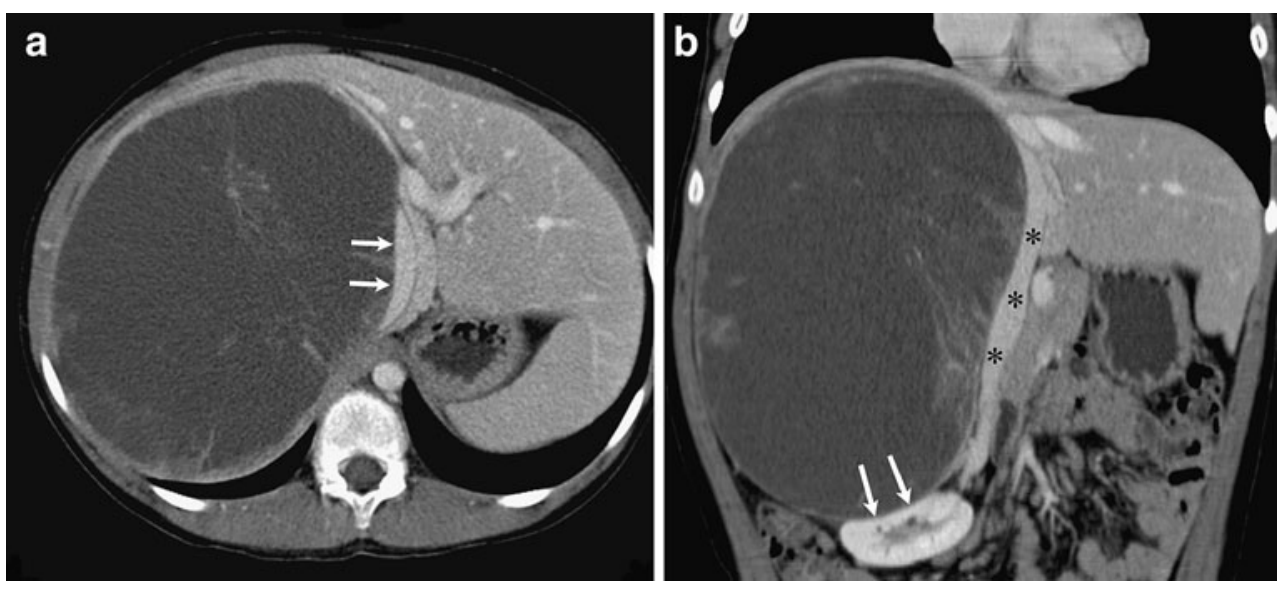

imaging, which should be justified by the probability of recurrence [20].

\section{MRI}

MRI is used increasingly for pediatric tumor imaging because of inherent advantages compared to other imaging modalities, including the absence of ionizing radiation and excellent soft-tissue contrast resolution. MRI has limitations when used to evaluate the lungs because of low proton density and high susceptibility effect. However, lung nodules of diameter larger than 3-4 $\mathrm{mm}$ can be detected with MRI at $1.5 \mathrm{~T}$ [21] (Fig. 6). In general, MRI is limited by long scan times and associated motion artifact. The combination of a state-of-the-art multichannel (e.g., 32channel) body-array coil and parallel imaging can reduce scan time without degrading images; it may in fact improve
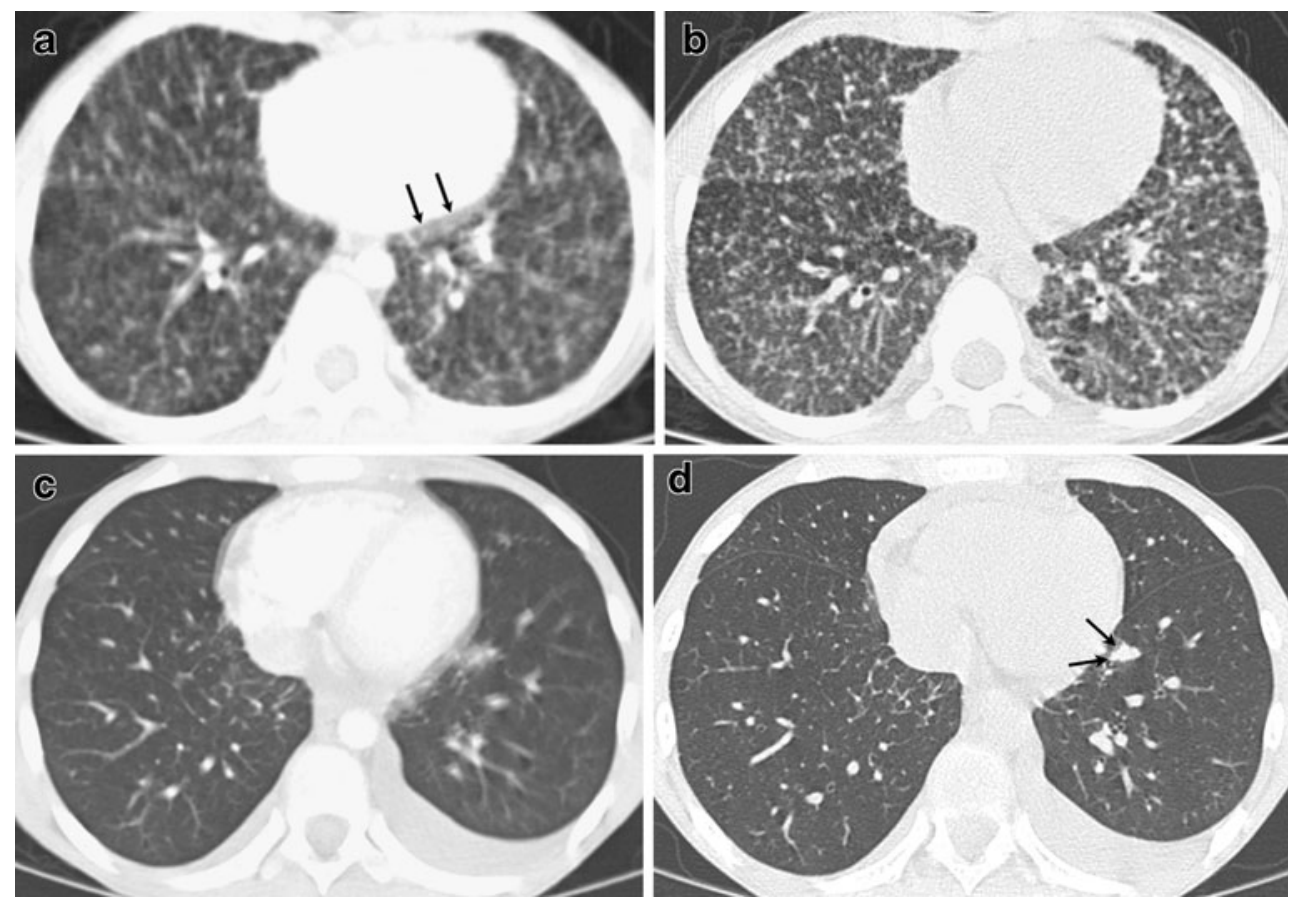

Fig. 4 a-d Reduction of motion artifacts on chest CT by partial sequential scanning with or without electrocardiogram (ECG) synchronization. a Axial chest CT image obtained by free-breathing multi-slice spiral scanning in a 5-year-old girl with papillary thyroid carcinoma and lung metastasis shows severe blurring of the anatomic details of the lungs because of respiratory motion artifacts, making evaluation for metastatic lung nodules difficult. Cardiac pulsation artifacts are also seen (arrows). b Using free-breathing partial sequential scanning in the same patient, there is reduction of motion

artifacts, facilitating the recognition of the miliary pattern of the lung metastasis. c Axial chest CT image obtained by breath-hold multi-slice spiral scanning in a 12-year-old boy with anaplastic large cell lymphoma shows motion artifacts attributable to cardiac pulsation in both paracardiac lung fields, and bilateral pleural effusions. d Employing a breath-hold ECG-triggered sequential scanning technique almost completely eliminates the cardiac pulsation artifacts, facilitating the identification of a nodular lymphoma lesion (arrows) in the anteromedial basal segment of the left lower lobe 
Fig. 5 a-d Use of prone chest $\mathrm{CT}$ to distinguish a lung nodule from subsegmental atelectasis. a Axial chest CT image taken in the supine position of a 9-yearold boy with anaplastic large cell lymphoma shows subpleural nodular opacity (arrow) in the dependent portion of the left lower lobe. b Prone CT image demonstrates disappearance of the subpleural nodular opacity indicating that the opacity seen on supine CT represented subsegmental atelectasis. c Axial chest $\mathrm{CT}$ image taken in the supine position of a 6-year-old boy with hepatoblastoma shows a lung nodule (arrow) in the right basal lung that needs to be distinguished from dependent opacity. d Prone CT image unequivocally reveals a tiny metastatic lung nodule (arrow)

Fig. 6 a, b Hepatoblastoma in a 9-year-old boy. a Axial chest CT image shows multiple metastatic lung nodules. b Coronal breath-hold T1-weighted turbo gradient-echo chest MR image (TR/TE, 5.7/2.8 ms; flip angle, $15^{\circ}$ ) reveals four metastatic nodules (arrows) in the right lung
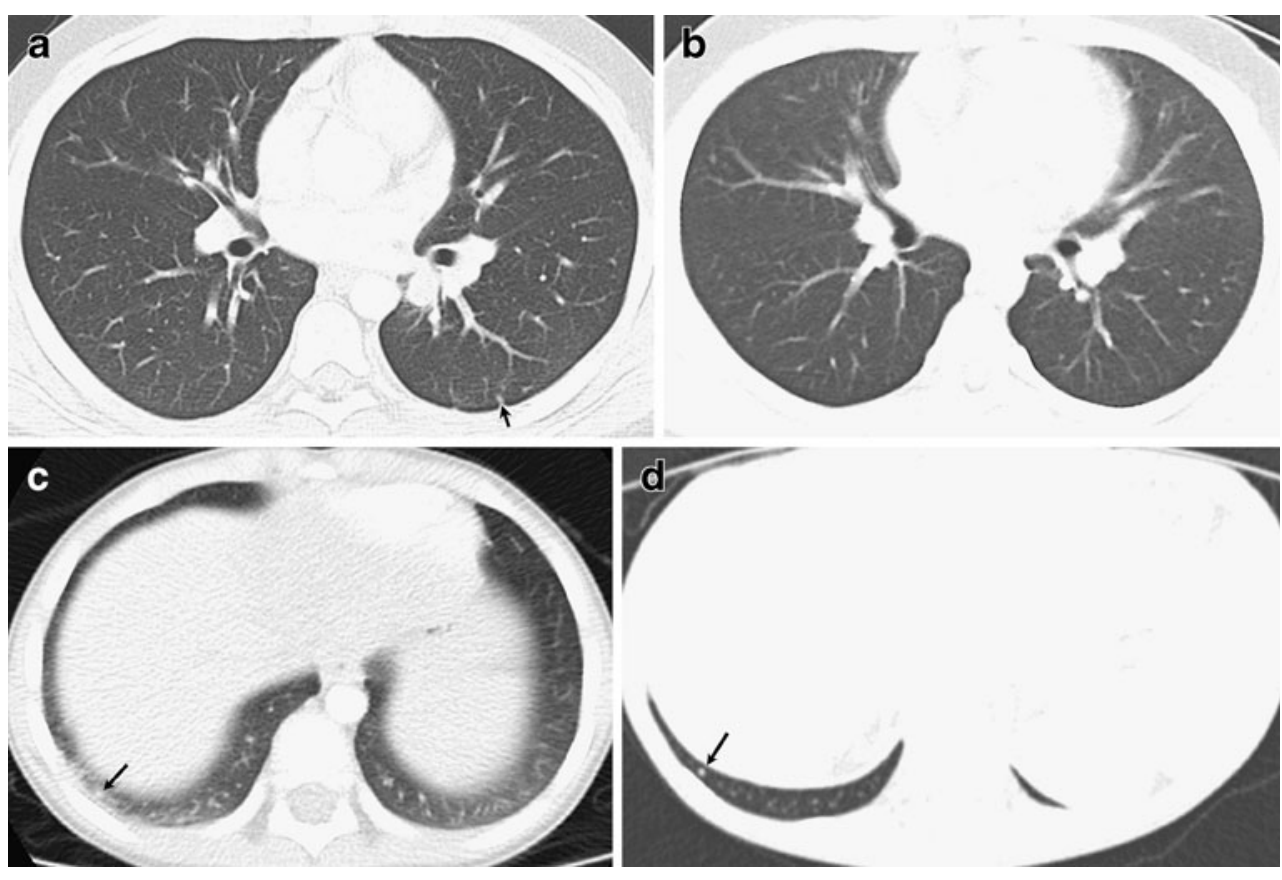
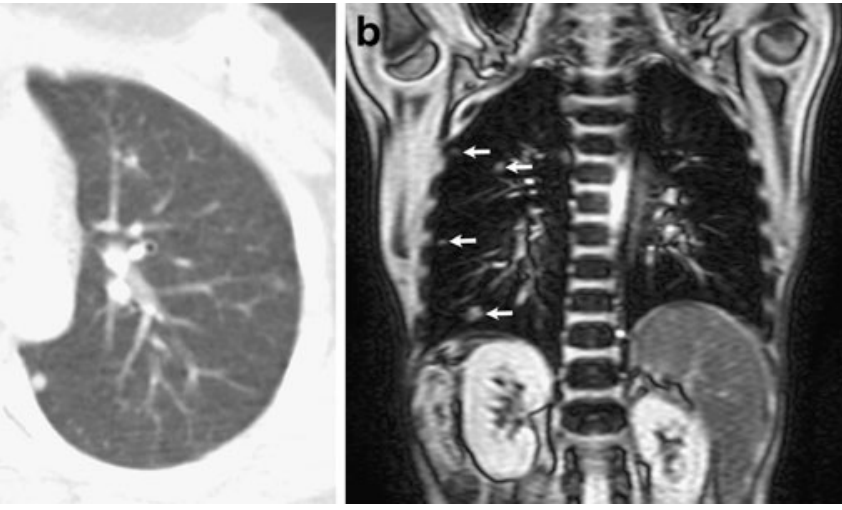

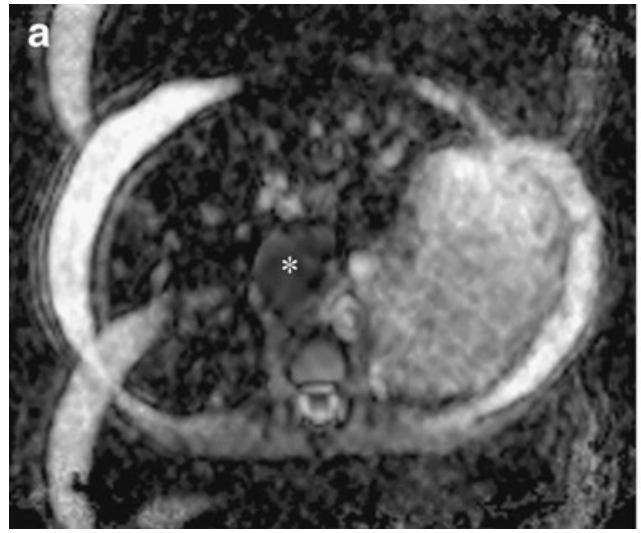

Fig. 7 a, b Neuroblastoma in an 8-year-old girl. a Respirationinduced ghosting artifacts are prominent and degrade the image quality of an apparent diffusion coefficient (ADC) map (b values of 0 and $800 \mathrm{~s} / \mathrm{mm}^{2}$ ) of the upper abdomen. b Presaturation bands placed

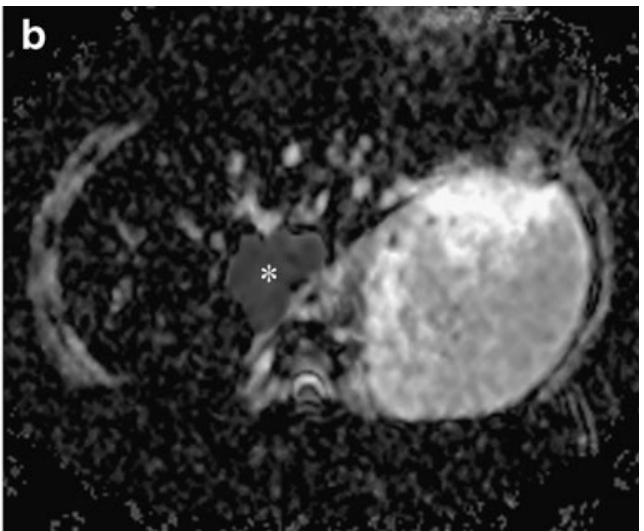

in the anterior and posterior body walls substantially reduce respiratory motion artifacts on a follow-up ADC map. Of note, a tumor (asterisk) with restricted water diffusion is seen in the caudate lobe of the liver 


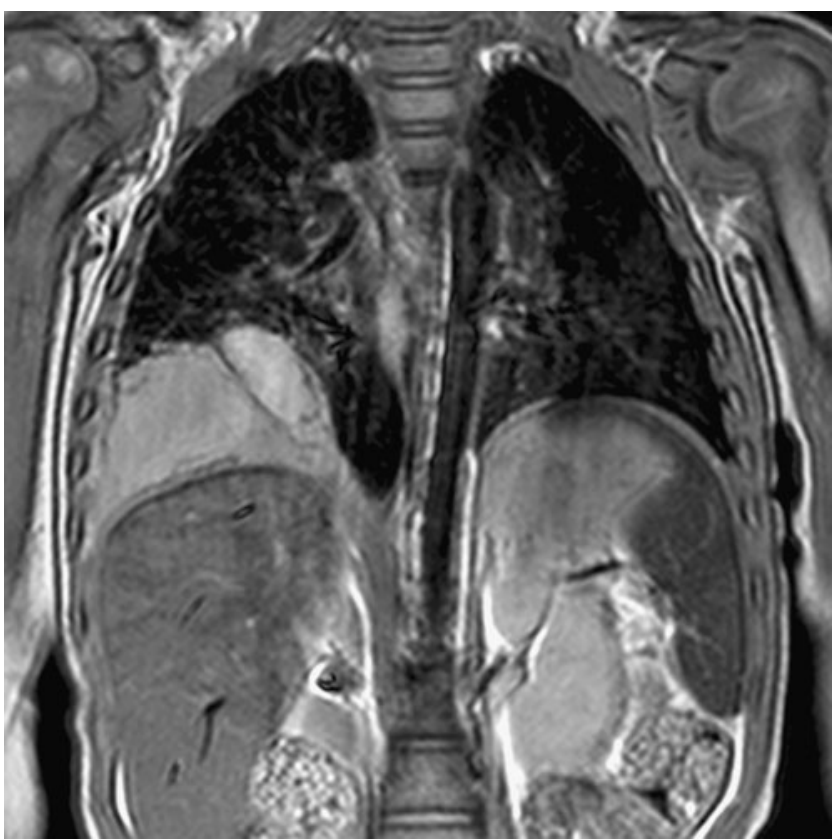

Fig. 9 Pleuropulmonary blastoma in a 2-year-old boy. Coronal ECGtriggered T1-weighted spin-echo echo-planar image (TR/TE, 437/ $15 \mathrm{~ms}$; flip angle, $90^{\circ}$ ) shows residual tumor after chemotherapy in the lower right thorax. Fortunately, the suspected lesion did not show tumor cells on histologic examination of the surgical specimen. An animation of the real-time chest MRI is provided online, and this demonstrates restricted motion of the lateral portion of the tumor due to adhesions

systemic fibrosis cannot be overemphasized, as pediatric oncologic patients are more likely to have impaired renal function secondary to anti-cancer therapy [34].

\section{Whole-body MRI}

In pediatric oncology, whole-body MRI offers great potential to provide critical information for initial and follow-up assessments [4, 35-45]. Coronal and sagittal short tau inversion recovery (STIR) images have been regarded as fundamental (Fig. 10). The total scan time for coronal and sagittal whole-body STIR is approximately 15$50 \mathrm{~min}$, depending on patient height. The STIR sequence, which is fairly insensitive to magnetic field inhomogeneity, provides excellent fat suppression in a large field-of-view, which is essential to obtain high-quality images. To ensure optimal image contrast, appropriate inversion times should be used (i.e., $150-160 \mathrm{~ms}$ at $1.5 \mathrm{~T}$ and $210-230 \mathrm{~ms}$ at $3.0 \mathrm{~T})[27,28,45]$. In fact, any pulse sequence may be used for whole-body MRI. However, the whole-body MRI protocol should be made acceptably short for children. Currently, $1.5 \mathrm{~T}$ offers better image quality than does $3.0 \mathrm{~T}$ for whole-body MRI. In addition to a quadrature body coil system, other coil systems can be employed to achieve high signal-to-noise ratios and high spatial resolution. The body-
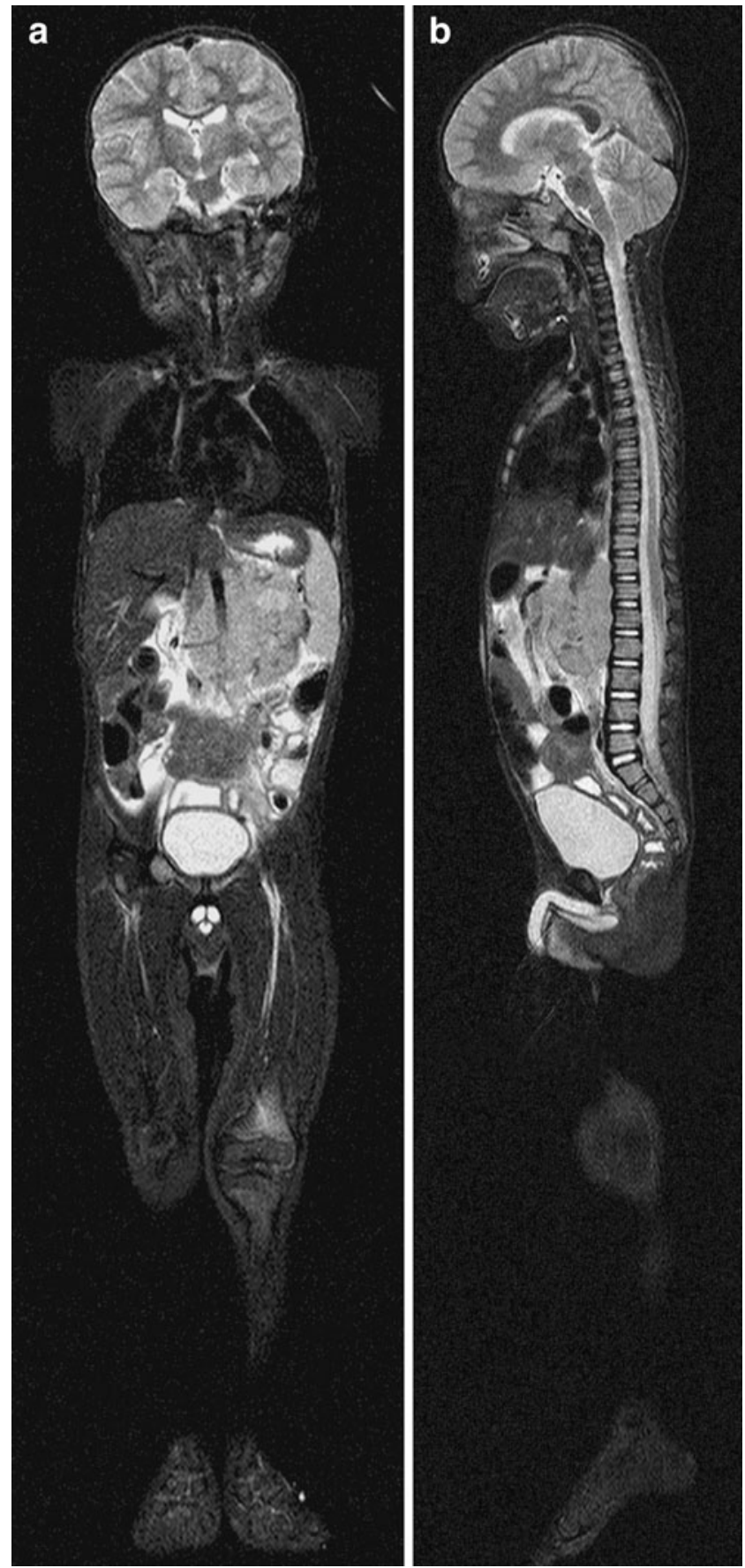

Fig. 10 a, b Neuroblastoma in a 2-year-old boy. Coronal (a) and sagittal (b) whole-body short tau inversion recovery (STIR) images (TR/TE/TI, 2,500/71/160 ms; flip angle, $90^{\circ}$ ) show a large, lobulated, para-aortic retroperitoneal solid tumor. Stitched images from several scan locations

size-adapted coil selection for whole-body MRI used at our institution is described in Table 1. Whole-body MRI has been shown to be consistently superior to bone scintigraphy in detecting bone metastases [4, 35, 40, 41], whereas whole-body MRI and PET-CT are complementary [41, 44]. 
Table 1 Suggested body-sizeadapted pediatric whole-body MRI coil selection

\begin{tabular}{llll}
\hline $\begin{array}{l}\text { Longitudinal } \\
\text { coverage }\end{array}$ & $\begin{array}{l}\text { Transverse } \\
\text { diameter }\end{array}$ & $\begin{array}{l}\text { Magnetic field } \\
\text { strength }\end{array}$ & Coil system \\
\hline Up to $95 \mathrm{~cm}$ & Up to $53 \mathrm{~cm}$ & $1.5 \mathrm{~T}$ & $\begin{array}{l}\text { Neurovascular and spine coil } \\
\text { Up to } 125 \mathrm{~cm}\end{array}$ \\
Up to $53 \mathrm{~cm}$ & $1.5 \mathrm{~T}$ & $\begin{array}{l}\text { Sliding 16-channel body-array coil } \\
\text { 32-channel total imaging matrix }\end{array}$ \\
Up to $180 \mathrm{~cm}$ & Up to $50 \mathrm{~cm}$ & $1.5 \mathrm{~T}$ & $\begin{array}{c}\text { technology } \\
\text { Quadrature body coil }\end{array}$ \\
Up to $190 \mathrm{~cm}$ & $\begin{array}{c}\text { Shorter than } \\
45 \mathrm{~cm}\end{array}$ & $3.0 \mathrm{~T}$ & Quadrature body coil \\
\hline
\end{tabular}

MRI is usually better for evaluating the brain, liver, and bone marrow, whereas PET-CT is generally better for lungs and the lymph nodes. Recently, whole-body diffusionweighted MRI was found to be useful to stage patients with lymphoma [46, 47]. This imaging technique is particularly promising in children because most pediatric malignant tumors are small round cell tumors with restricted water diffusion (Fig. 11). In contrast, diffusion-weighted MRI may not be useful for evaluating spindle cell tumors or Langerhans cell histiocytosis [4].

\section{Conclusion}

Pediatric oncologic imaging protocols should be individually optimized to achieve the shortest examination time, the
Fig. 11 a, b Burkitt lymphoma in a 10 -year-old boy. Wholebody diffusion-weighted MRI (DWI) (a) and fluorodeoxyglucose-positron emission tomography (FDGPET) (b) show extensive involvement of the lungs, liver, both kidneys, and almost the entire skeleton. Compared with FDG-PET imaging, DWI delineates anatomic details more precisely because of higher spatial resolution. The normal testicles (arrows) are hyperintense on DWI. High activity in the urinary bladder (asterisk) on FDG-PET may obscure a pelvic lesion

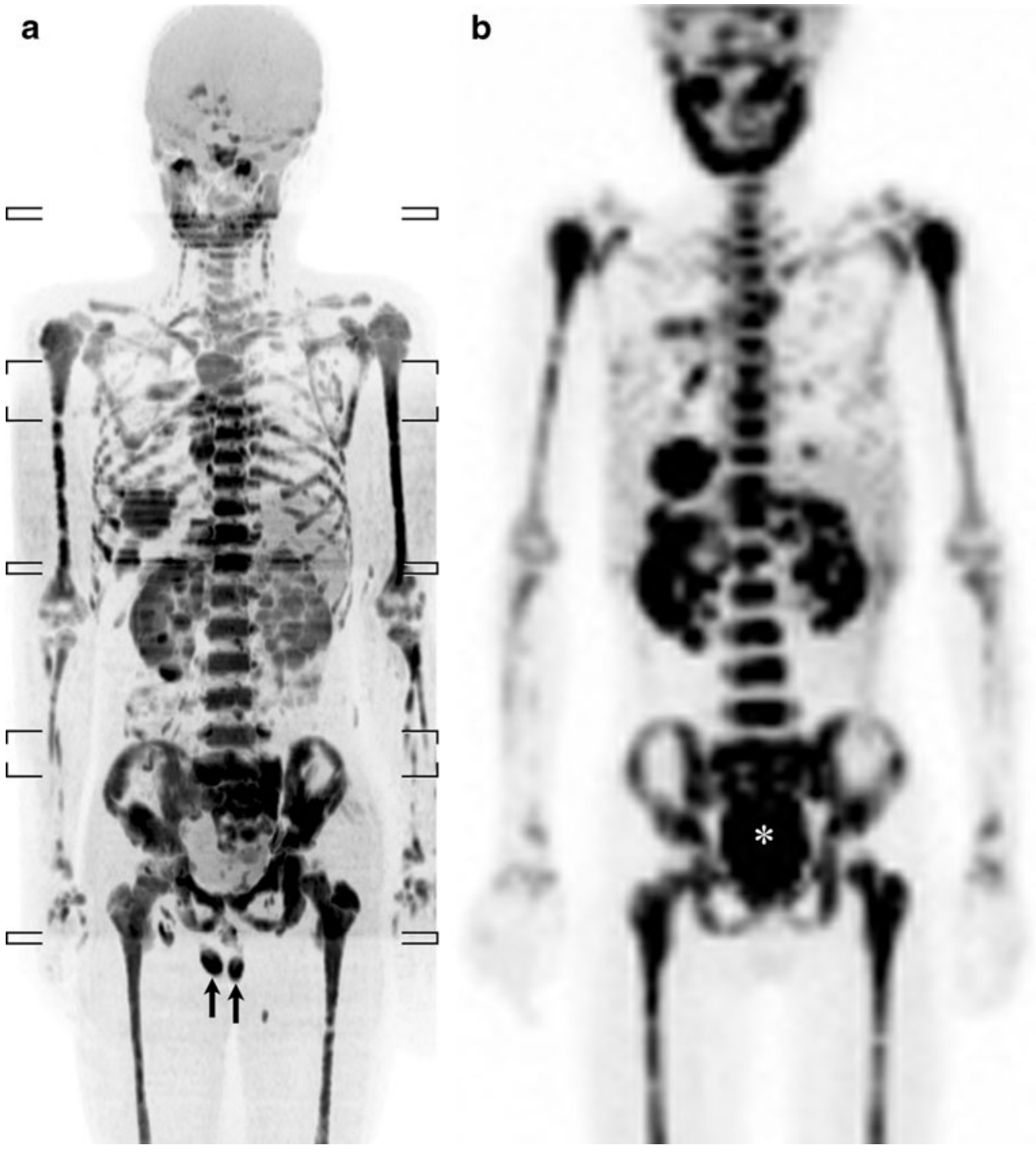


highest image quality, the lowest risks of radiation exposure and other adverse effects, and the maximum clinical benefits. Moreover, every effort should be made to eliminate redundant examinations, which are unnecessary burdens to the child. In this regard, bone scintigraphy may be omitted when another superior imaging modality is available. CT and scintigraphy should be used cautiously in children due to ionizing radiation exposure. Whole-body MRI and PET-CT may become the mainstays of pediatric tumor imaging.

\section{References}

1. Kucera ED, Karmazyn B, Cohen MD et al (1997) Imaging modalities in pediatric oncology. Radiol Clin North Am 35:1281-1300

2. McCarville MB (2008) New frontiers in pediatric oncologic imaging. Cancer Imaging 8:87-92

3. Azouz EM, Saigal G, Rodriguez MM et al (2005) Langerhans cell histiocytosis: pathology, imaging and treatment of skeletal involvement. Pediatr Radiol 35:103-115

4. Goo HW, Yang DH, Ra YS et al (2006) Whole-body MRI of Langerhans cell histiocytosis: comparison with radiography and bone scintigraphy. Pediatr Radiol 36:1019-1031

5. Lim JH, Ko YT, Lee DH (1990) Sonographic sliding sign in localization of right upper quadrant mass. J Ultrasound Med 9:455-459

6. Lyshchik A, Drozd V, Schloegl S et al (2004) Three-dimensional ultrasonography for volume measurement of thyroid nodules in children. J Ultrasound Med 23:247-254

7. Kim SH, Choi BI, Kim KW et al (2003) Extended field-of-view sonography: advantages in abdominal applications. J Ultrasound Med 22:385-394

8. Yerli H, Eksioglu SY (2009) Extended field-of-view sonography: evaluation of the superficial lesions. Can Assoc Radiol J 60:35-39

9. Kim JH, Goo HW, Yoon CH (2003) Intra-abdominal desmoplastic small round-cell tumour: multiphase CT findings in two children. Pediatr Radiol 33:418-421

10. Chooi WK, Matthews S, Bull MJ (2005) Multislice computed tomography in staging lung cancer: the role of multiplanar image reconstruction. J Comput Assist Tomogr 29:357-360

11. Sandrasegaran K, Rydgerg J, Tann M et al (2007) Benefits of routine use of coronal and sagittal reformations in multi-slice CT examination of the abdomen and pelvis. Clin Radiol 62:340-347

12. Ha HI, Goo HW, Seo JB et al (2006) Effects of high-resolution $\mathrm{CT}$ of the lung using partial versus full reconstruction on motion artifacts and image noise. AJR Am J Roentgenol 187:618-622

13. Pauls S, Aschoff AJ, Wahl J et al (2005) Multi-detector row CT: is prospective electrocardiographic triggering improving the detection of small pulmonary tumors? Acad Radiol 12:614-619

14. Goo HW (2010) State-of-the art CT imaging techniques for congenital heart disease. Korean J Radiol 11:4-18

15. McCarville MB, Lederman HM, Santana VM et al (2006) Distinguishing benign from malignant pulmonary nodules with helical chest CT in children with malignant solid tumors. Radiology 239:514-520

16. Silva CT, Amaral JG, Moineddin R et al (2010) CT characteristics of lung nodules present at diagnosis of extrapulmonary malignancy in children. AJR Am J Roentgenol 194:772-778

17. Goo JM, Chung MJ, Lee HJ et al (2003) Posterior subpleural nodules in patients with underlying malignancies: value of prone computed tomography. J Comput Assist Tomogr 27:274-278
18. Yang DH, Goo HW (2008) Pediatric 16-slice CT protocol: radiation dose and image quality. J Korean Radiol Soc 59:333347

19. Nievelstein RA, van Dam IM, van der Molen AJ (2010) Multidetector CT in children: current concepts and dose reduction strategies. Pediatr Radiol 40:1324-1344

20. Chong AL, Grant RM, Ahmed BA et al (2010) Imaging in pediatric patients: time to think again about surveillance. Pediatr Blood Cancer 55:407-413

21. Biederer J, Hintze C, Fabel M (2008) MRI of pulmonary nodules: technique and diagnostic value. Cancer Imaging 19:125-130

22. Henzier T, Dietrich O, Krissak R et al (2009) Half-Fourieracquisition single-shot turbo spin-echo (HASTE) MRI of the lung at 3 Tesla using parallel imaging with 32-receiver channel technology. J Magn Reson Imaging 30:541-546

23. Chung T, Muthupillai R (2004) Application of SENSE in clinical pediatric body MR imaging. Top Magn Reson Imaging 15:187196

24. Tang Y, Yamashita Y, Namimoto T et al (1998) Characterization of focal liver lesions with half-fourier acquisition single-shot turbo-spin-echo (HASTE) and inversion recovery (IR)-HASTE sequences. J Magn Reson Imaging 8:438-445

25. Vasanawala SS, Iwadate Y, Church DG et al (2010) Navigated abdominal T1-W MRI permits free-breathing image acquisition with less motion artifacts. Pediatr Radiol 40:340-344

26. Hirokawa Y, Isoda H, Maetani YS et al (2008) Evaluation of motion correction effect and image quality with the periodically rotated overlapping parallel lines with enhanced reconstruction (PROPELLER) (BLADE) and parallel imaging acquisition technique in the upper abdomen. J Magn Reson Imaging 28:957-962

27. Chavhan GB, Babyn PS, Singh M et al (2009) MR imaging at 3.0 $\mathrm{T}$ in children: technical differences, safety issues, and initial experience. Radiographics 29:1451-1466

28. Goo HW (2010) High field strength magnetic resonance imaging in children. J Korean Med Assoc 53:1093-1102

29. Willinek WA, Gieseke J, Kukuk GM et al (2010) Dual-source parallel radiofrequency excitation body MR imaging compared with standard MR imaging at $3.0 \mathrm{~T}$ : initial clinical experience. Radiology 256:966-975

30. Zha Y, Li M, Yang J (2010) Dynamic contrast enhanced magnetic resonance imaging of diffuse spinal bone marrow infiltration in patients with hematological malignancies. Korean J Radiol 11:187-194

31. Padhani AR, Liu G, Koh DM et al (2009) Diffusion-weighted magnetic resonance imaging as a cancer biomarker: consensus and recommendations. Neoplasia 11:102-125

32. Alibek S, Cavallaro A, Aplas A et al (2009) Diffusion weighted imaging of pediatric and adolescent malignancies with regard to detection and delineation: initial experience. Acad Radiol 16:866871

33. Lee CH, Goo JM, Kim YT et al (2010) The clinical feasibility of using non-breath-hold real-time MR-echo imaging for the evaluation of mediastinal and chest wall tumor invasion. Korean $\mathrm{J}$ Radiol 11:37-45

34. Juluru K, Vogel-Claussen J, Macura KJ et al (2009) MR imaging in patients at risk for developing nephrogenic systemic fibrosis: protocols, practices, and imaging techniques to maximize patient safety. Radiographics 29:9-22

35. Daldrup-Link HE, Franzius C, Link TM et al (2001) Whole-body MR imaging for detection of bone metastases in children and young adults: comparison with skeletal scintigraphy and FDG PET. AJR Am J Roentgenol 177:229-236

36. Mazumdar A, Siegel MJ, Narra V et al (2002) Whole-body fast inversion recovery $\mathrm{MR}$ imaging of small cell neoplasms in pediatric patients: a pilot study. AJR Am J Roentgenol 179:1261-1266 
37. Laffan EE, O'Connor R, Ryan SP et al (2004) Whole-body magnetic resonance imaging: a useful additional sequence in paediatric imaging. Pediatr Radiol 34:472-480

38. Kellenberger CJ, Epelman M, Miller SF et al (2004) Fast STIR whole-body MR imaging in children. Radiographics 24:1317-1330

39. Kellenberger CJ, Miller SF, Khan M et al (2004) Initial experience with FSE STIR whole-body MR imaging for staging lymphoma in children. Eur Radiol 14:1829-1841

40. Goo HW, Choi SH, Ghim T et al (2005) Whole-body MRI of paediatric malignant tumours: comparison with conventional oncologic imaging methods. Pediatr Radiol 35:766-773

41. Kumar J, Seith A, Kumar A et al (2008) Whole-body MR imaging with the use of parallel imaging for detection of skeletal metastases in pediatric patients with small-cell neoplasms: comparison with skeletal scintigraphy and FDG PET/CT. Pediatr Radiol 38:953-962

42. Lim GY, Hahn ST, Chung NG et al (2009) Subcutaneous panniculitis-like T-cell lymphoma in a child: whole-body MRI in the initial and follow-up evaluations. Pediatr Radiol 39:5761

43. Krohmer S, Sorge I, Krausse A et al (2010) Whole-body MRI for primary evaluation of malignant disease in children. Eur J Radiol $74: 256-261$

44. Punwani S, Taylor SA, Bainbridge A et al (2010) Pediatric and adolescent lymphoma: comparison of whole-body STIR halfFourier RARE MR imaging with an enhanced PET/CT reference for initial staging. Radiology 255:182-190

45. Goo HW (2010) Whole-body MRI of neuroblastoma. Eur J Radiol 75:306-314

46. Lin C, Luciani A, Itti E et al (2010) Whole-body diffusionweighted magnetic resonance imaging with apparent diffusion coefficient mapping for staging patients with diffuse large B-cell lymphoma. Eur Radiol 20:2027-2038

47. Kwee TC, Takahara T, Vermoolen MA et al (2010) Whole-body diffusion-weighted imaging for staging malignant lymphoma in children. Pediatr Radiol 40:1592-1602 